\title{
Development of antigen-specific ELISA for circulating autoantibodies to extracellular matrix protein 1 in lichen sclerosus
}

\author{
Noritaka Oyama, 1,2,3 Ien Chan, ${ }^{1}$ Sallie M. Neill, ${ }^{2}$ Andrew P. South, ${ }^{1}$ Fenella Wojnarowska, ${ }^{4}$ \\ Yoshio Kawakami, ${ }^{3}$ David D'Cruz, ${ }^{5}$ Kirti Mepani, ${ }^{5}$ Graham J. Hughes, ${ }^{5}$ Balbir S. Bhogal, ${ }^{2}$ \\ Fumio Kaneko, ${ }^{3}$ Martin M. Black, ${ }^{2}$ and John A. McGrath'1 \\ ${ }^{1}$ Genetic Skin Disease Group, and 2Department of Immunofluorescence, St. John's Institute of Dermatology, The Guy's, King's College and \\ St. Thomas' Hospitals' Medical School, St. Thomas' Hospital, London, United Kingdom. '3epartment of Dermatology, Fukushima Medical University \\ School of Medicine, Fukushima, Japan. “Department of Dermatology, The Churchill Hospital, Oxford, United Kingdom. ${ }^{5}$ Lupus Research Unit, \\ Rayne Institute, Immunology Department, St. Thomas' Hospital, London, United Kingdom.
}

\begin{abstract}
Lichen sclerosus is a common, acquired chronic inflammatory skin disease of unknown etiology, although circulating autoantibodies to the glycoprotein extracellular matrix protein 1 (ECM1) have been detected in most patients' sera. We have examined the nature of ECM1 epitopes in lichen sclerosus sera, developed an ELISA system for serologic diagnosis, and assessed clinicopathological correlation between ELISA titer and disease. Epitope-mapping studies revealed that lichen sclerosus sera most frequently recognized the distal second tandem repeat domain and carboxyl-terminus of ECM1. We analyzed serum autoantibody reactivity against this immunodominant epitope in 413 individuals (95 subjects with lichen sclerosus, 161 normal control subjects, and 157 subjects with other autoimmune basement membrane or sclerosing diseases). The ELISA assay was highly sensitive; 76 of 95 lichen sclerosus patients $\mathbf{( 8 0 . 0 \% )}$ exhibited IgG reactivity. It was also highly specific $(\mathbf{9 3 . 7 \% )}$ in discriminating between lichen sclerosus and other disease/control sera. Higher anti-ECM1 titers also correlated with more longstanding and refractory disease and cases complicated by squamous cell carcinoma. Furthermore, passive transfer of affinity-purified patient IgG reproduced some histologic and immunopathologic features of lichen sclerosus skin. This new ELISA is valuable for the accurate detection and quantification of anti-ECM1 autoantibodies. Moreover, the values may have clinical significance in patients with lichen sclerosus.
\end{abstract}

\section{Introduction}

Lichen sclerosus is a common acquired skin disorder (1-3). The typical clinical presentation consists of pale, indurated papules and plaques with intractable irritation. Any skin may be involved, but the most common site is the genitalia ( $85 \%$ to $98 \%$ of cases), although disease affecting the head, neck, scalp, palms and soles, area around the eye, tongue, and area around stoma sites has been reported (1,3-5). Clinical and epidemiological studies suggest that the condition is underreported and may have a prevalence of more than 1 in $300(2,5)$. Females are more commonly affected than males; the ratio of female to male patients is as high as 10:1. Lichen sclerosus can occur at any age, but a bimodal peak occurs in prepubertal children and then in postmenopausal women and in men 30 to 50 years of age $(1,3)$.

The disease may be difficult to diagnose. It is sometimes mistaken for signs of child abuse in young children or for an inflammatory disorder such as lichen planus in adults. Currently no specific disease markers or diagnostic tests other than a skin biopsy identify this disease, although even a skin biopsy may show overlap with other inflammatory skin diseases. Furthermore, the

Nonstandard abbreviations used: arbitrary unit (AU); area under the curve (AUC); confidence interval (CI); extracellular matrix protein 1 (ECM1); glutathione

$S$-transferase (GST); receiver operating characteristic (ROC); Tris-buffered saline (TBS).

Conflict of interest: The authors have declared that no conflict of interest exists.

Citation for this article: J. Clin. Invest. 113:1550-1559 (2004).

doi:10.1172/JCI200420373. clinical course may be protracted with only limited symptomatic relief from the topical application of high-dose fluorinated corticosteroids. Rarely, systemic immunosuppression (e.g., with oral cyclosporin) may be required for severe disease (6). Genital disease may also result in severe scarring, which leads to difficulty in micturition and to impaired sexual activity. Some cases may also be complicated by malignancy $(\sim 5 \%)$, most frequently squamous cell carcinoma $(2,3)$. Thus, lichen sclerosus represents an important disease process to investigate.

The etiology of lichen sclerosus is uncertain, but accumulating evidence suggests an autoimmune basis to the disorder. For example, some reports have described a disease association with HLA class II antigen DQ7-DQ9 $(7,8)$, as well as an increased incidence of other autoimmune diseases and autoantibodies in some patients. Associated disorders include thyroid disease, pernicious anemia, diabetes mellitus, alopecia areata, vitiligo, and mucous membrane pemphigoid $(1,4,9)$. Historically, data implicating a humoral autoimmune response in lichen sclerosus have been limited to a single 90-year-old case report of probable lichen sclerosus being induced by injection of serum from an affected individual into nonlesional skin (10). However, we have recently identified specific autoantibodies to a particular skin antigen, extracellular matrix protein 1 (ECM1), in sera from the majority of patients with lichen sclerosus (11). These data provide preliminary clues to unraveling the lichen sclerosus disease mechanism.

ECM1 is present within epidermis and dermis and has been implicated in aspects of epidermal differentiation and in protein- 


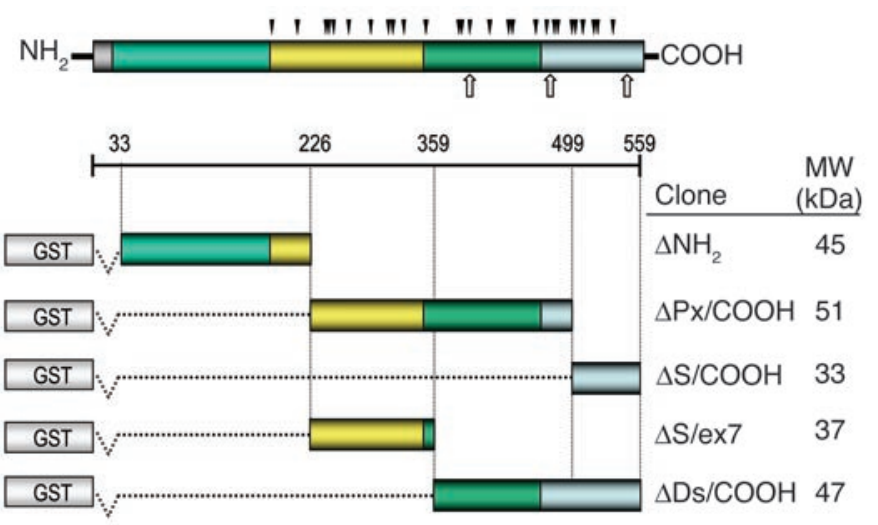

\section{Figure 1}

Schematic representation of ECM1 protein and its five recombinant GST fusion fragments. The distinct molecular domains comprise a signal peptide, a cysteine-free $\mathrm{NH}_{2}$-terminal domain, two tandem repeat domains, and a $\mathrm{COOH}$-terminal domain. The position of the single cysteine residues and potential $\mathrm{N}$-glycosylation sites are indicated. The full-length 1.8-kb ECM1 cDNA was initially divided into three different fragments, $\Delta \mathrm{NH}_{2}$ (33 to 226 amino acids), $\Delta \mathrm{Px} / \mathrm{COOH}$ (226 to 499 amino acids), and $\Delta \mathrm{S} / \mathrm{COOH}$ (499 to 559 amino acids), designed to span almost the entire ECM1. Two further fragments were then synthesized. The $\Delta S / e x 7$ fragment encodes part of the $\Delta \mathrm{Px} / \mathrm{COOH}$ domain (226 to 359 amino acids), and the $\Delta \mathrm{Ds} / \mathrm{COOH}$ fragment encodes the distal part of the second tandem repeat domain and $\mathrm{COOH}$-terminus (359 to 559 amino acids). All fragments were fused with GST-encoded cDNA at the $\mathrm{NH}_{2}$-terminus of the recombinant proteins. The amino acid residue numbers are shown as described elsewhere (13). The predicted molecular weights $(\mathrm{kDa})$ of each recombinant fusion fragment are shown on the right.
As assessed by Coomassie gel staining and immunoblotting with anti-glutathione $S$-transferase (anti-GST) antibody, the GST tagging at the $\mathrm{NH}_{2}$-terminus of the recombinant proteins allowed for efficient purification of all five recombinants in a single step (Figure 2). Each of these recombinant proteins was detected at the predicted molecular mass, as a doublet band, with the exception of multiple bands for $\Delta \mathrm{S} / \mathrm{COOH}$. The bands with a lower molecular mass probably result from degradation products during sample preparation (e.g., after protein extraction or SDS treatment) or from aberrant migration in SDS-gel electrophoresis. Each recombinant protein was finally eluted with more than $90 \%$ purity and sufficient protein concentration $(\sim 100 \mu \mathrm{g} / \mathrm{ml})$ for subsequent experiments.

IgG autoantibodies from lichen sclerosus sera react predominantly with the distal COOH-terminal region of ECM1.

protein interactions within the dermis $(12,13)$. The rationale for ECM1 as a target antigen in lichen sclerosus is supported by the recent identification of pathogenic mutations in the ECM1 gene in an inherited autosomal recessive skin disorder, lipoid proteinosis (OMIM 247100) (14). Histology of skin in this genetic condition shows considerable overlap with skin biopsy findings in lichen sclerosus, including disruption of basement membrane and hyaline (glassy) changes in the upper dermis $(11,14,15)$. From a clinicopathologic perspective, disruption of ECM1 function by acquired autoantibodies or inherited gene mutations appears to contribute significantly to the disease pathology in lichen sclerosus or lipoid proteinosis, respectively. Further characterization of ECM1 antibodies in lichen sclerosus may, therefore, give new insight into this common acquired condition.

The aim of this study was to characterize the antigenic epitopes within ECM1 targeted by autoantibodies in sera from patients with lichen sclerosus. A further goal was to develop a sensitive and quantitative ELISA specific for autoantibodies directed against ECM1. We applied this ELISA in a clinical context, to determine its potential utility in the serologic diagnosis of lichen sclerosus and to assess whether any clinicopathological correlation exists between the ELISA results and the clinical status of individuals with lichen sclerosus.

\section{Results}

Generation of recombinant ECM1 fragments for epitope mapping. We have recently shown that the majority of lichen sclerosus patients' sera (74\%) contain IgG reactivity to the three isoforms of the native ECM1 protein (ECM1a, ECM1b, and ECM1c), as well as its full-length recombinant protein (11). To more rigorously identify antigenic epitopes within ECM1, we generated a series of bacterial recombinant fragments that cover almost the entire ECM1 sequence (Figure 1).
We tested IgG reactivity of 39 lichen sclerosus sera against the newly generated ECM1 recombinant fragments by immunoblotting. In this part of the study, five normal and six disease sera (three systemic lupus erythematosus and three bullous pemphigoid) were included as control subjects. The results demonstrated that lichen sclerosus autoantibodies recognize multiple antigenic sites located throughout the ECM1 molecule (Figure 3 and Table 1).

Among the sera samples from the 39 patients, $16(41.0 \%)$ reacted with $\Delta \mathrm{NH}_{2}, 22(56.4 \%)$ reacted with $\Delta \mathrm{Px} / \mathrm{COOH}$, and four reacted with $(10.3 \%)$ with $\Delta \mathrm{S} / \mathrm{COOH}$. Interestingly, all the sera that reacted with $\Delta \mathrm{NH}_{2}$ also showed reactivity to $\Delta \mathrm{Px} / \mathrm{COOH}$, which suggests a heterogeneous reactivity to ECM1 in lichen sclerosus sera. Thus, this initial screen suggested that the $\triangle \mathrm{Px} / \mathrm{COOH}$ fragment was targeted most frequently.

To further characterize the highest immunoreactivity against the $\Delta \mathrm{Px} / \mathrm{COOH}$, we generated an additional fragment, $\Delta \mathrm{S} / \mathrm{ex} 7$, which overlaps with the $\mathrm{NH}_{2}$-terminal by 133 amino acids of $\Delta \mathrm{Px} / \mathrm{COOH}$ (Figure 1). Repeat immunoblotting with the $22 \Delta \mathrm{Px} / \mathrm{COOH}$-positive lichen sclerosus sera revealed that only one of these original indicate that the immunoreactive part of clone $\Delta \mathrm{Px} / \mathrm{COOH}$ lies between amino acids 359 and 499.

Combined with the other results that showed more than $10 \%$ of lichen sclerosus sera react with the COOH-terminal end of ECM1 $(\Delta \mathrm{S} / \mathrm{COOH})$, evidence indicated that the main immunodominant region of ECM1 was located between amino acids 359 and 559. This interpretation also takes note of the fact that all the sera that reacted with the $\Delta \mathrm{NH}_{2}$ clone also reacted with the indicated region.

To further define the immunodominant region, we generated 559 (Figure 1) and repeated the immunoblotting with the abovementioned sera (Figure 3 and Table 1). As expected, specific immunoreactivity was detected in the majority of lichen sclerosera showed any reactivity (albeit faint) to $\Delta \mathrm{S} / \mathrm{ex} 7$. These data an extra fragment $(\Delta \mathrm{Ds} / \mathrm{COOH})$ specific for amino acids 359 to 


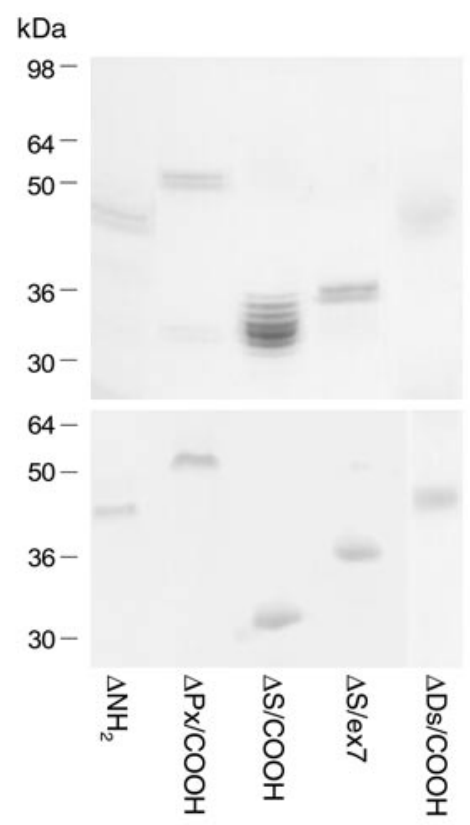

Figure 2

Purification of recombinant ECM1-GST fusion proteins. The purified ECM1-GST fusion recombinant proteins were separated on SDS-polyacryl amide gel and detected by Coomassie blue staining (top). The identical gel was immunoblotted using anti-GST antibody (bottom). Molecular weights are indicated on the left column.

sus sera $(28 / 39,71.8 \%)$. Almost all the sera that were positive on the initial immunoblotting when any of other ECM1 recombinant fragments were used remained positive when tested with $\Delta \mathrm{Ds} / \mathrm{COOH}$. None of the normal or disease control sera showed reactivity to any of the ECM1 recombinants. These results suggest that the distal second tandem repeat and $\mathrm{COOH}$-terminal region encompassing amino acids 359 to 559 can detect almost the entire spectrum of antibody reactivity against ECM1 in patients' sera.

Lichen sclerosus antibodies are directed against specific, but not cross-reactive, ECM1 epitopes. To determine the epitope specificity of lichen sclerosus antibodies, we performed indirect immunofluorescence on normal human skin sections. However, immunostaining with standard dilutions of the sera was negative in almost all cases, as demonstrated previously (11). This finding suggests that the binding affinity or the titer of the antibodies is extremely low. The latter assumption is supported by evidence that any dilution of the patients' sera greater than 1:20 resulted in a loss of the specific bands when reassessed by recombinant protein immunoblotting (data not shown).

We therefore affinity-purified the IgG fractions from six representative lichen sclerosus sera that were positive on immunoblotting with recombinant proteins (selected sera comprised three that were reactive with $\Delta \mathrm{Ds} / \mathrm{COOH}$ alone and three others that were reactive with both $\Delta \mathrm{NH}_{2}$ and $\left.\Delta \mathrm{Ds} / \mathrm{COOH}\right)$.

Immunofluorescence staining of normal human skin with the affinity-purified IgG antibodies from all patients' sera displayed an intracellular signal in basal keratinocytes; weaker staining occurred in the suprabasal keratinocytes and around dermal blood vessels (Figure 4B). This immunolabeling appearance was very similar to the staining pattern seen with anti-ECM1 rabbit polyclonal antibody (Figure 4A). The positive staining with the
$\Delta \mathrm{Ds} / \mathrm{COOH}$-reactive sera was blocked efficiently by preabsorption with excess amounts of the $\Delta \mathrm{Ds} / \mathrm{COOH}$ recombinant protein (Figure $4 \mathrm{C}$ ). The positive staining with a dual $\Delta \mathrm{NH}_{2}$-reactive and $\Delta \mathrm{Ds} / \mathrm{COOH}$-reactive sera was not altered or was only slightly diminished by preabsorption with either $\Delta \mathrm{NH}_{2}$ or $\Delta \mathrm{Ds} / \mathrm{COOH}$ recombinants separately (Figure 4D), but was blocked almost completely when preabsorbed with a mixture of $\Delta \mathrm{NH}_{2}$ and $\Delta \mathrm{Ds} /$ $\mathrm{COOH}$ recombinants together (Figure $4 \mathrm{E}$ ). None of the control recombinant proteins, GST alone or BP180 NC16a, altered the positive staining with the affinity purified sera (Figure 4F). Immunolabeling did not occur with affinity-purified normal human control sera (data not shown). These findings indicate that immunoreactivity of the affinity-purified IgGs from lichen sclerosus is specific for ECM1, and no antibody cross-reactivity against other epitopes within ECM1 occurs.

A novel ELISA using the distal second tandem repeat and $\mathrm{COOH}$-terminal region of ECM1 detects IgG reactivity in lichen sclerosus patients' sera. Sera from patients with lichen sclerosus $(n=95)$ and, as controls, from normal volunteers $(n=161)$, systemic lupus erythematosus patients $(n=70)$, bullous pemphigoid patients $(n=72)$, and systemic sclerosis patients $(n=15)$ were tested for IgG reactivity against the most immunodominant $\Delta \mathrm{Ds} / \mathrm{COOH}$ fragment, immobilized onto an ELISA plate. The results were expressed as arbitrary units (AU) based on the positive and negative reference sera (1.0 and 0 OD values in the assay, respectively).

All the sera investigated by use of this ELISA exhibited AU ranging from 0 to 1.150 . Based on the maximization of the Youden index $(\mathrm{J}=$ sensitivity + specificity- 1$)$, the cutoff value for the ELISA was established at " 0.328 AU," which corresponds to a Youden index of 0.77 . The receiver operating characteristic (ROC) analysis revealed that the diagnostic accuracy of the ECM1-ELISA for lichen sclerosus, as estimated by the area under the curve (AUC), was 0.919 (95\% confidence interval [CI] 0.864 to 0.961 ) (Figure 5, inset). The mean intraplate and interplate coefficients of variation for the positive reference serum were $3.2 \%$ and $3.8 \%$, respectively (those for the negative reference serum were 1.0\% and 3.4\%).

When the selected cutoff value was used, 76 of 95 lichen sclerosus sera showed specific reactivity with the $\Delta \mathrm{Ds} / \mathrm{COOH}$, which results in a specificity and sensitivity of $93.7 \%$ and $80.0 \%$, respectively (Figure 5). Only three sera (3.4\%) yielded marginal OD readings. A linear correlation in the immunoreactivity against the $\triangle \mathrm{Ds} / \mathrm{COOH}$ occurred between immunoblotting and ELISA, although the detection sensitivity in lichen sclerosus sera was higher by ELISA than by immunoblotting when the full-length ECM1 recombinant protein was used (11).

In contrast, comparative control groups did not show any significant reactivity to the $\Delta \mathrm{Ds} / \mathrm{COOH}$ recombinant protein (Table 2). Of 161 normal, healthy control subjects, 7 (4.3\%; 95\% CI 1 to 7 ) had

\section{Table 1}

Lichen sclerosus immunoreactivity against a series of ECM1-GST recombinant proteins on immunoblotting

$\begin{array}{lc}\text { Clone } & \text { Reactivity of lichen sclerosus sera }(\boldsymbol{n}=\mathbf{3 9}) \\ \Delta \mathrm{NH}_{2} & 16(41.0 \%) \\ \Delta \mathrm{Px} / \mathrm{COOH} & 22(56.4 \%) \\ \Delta \mathrm{S} / \mathrm{COOH} & 4(10.3 \%) \\ \Delta \mathrm{S} / \mathrm{ex} 7 & 1(2.6 \%) \\ \Delta \mathrm{DS} / \mathrm{COOH} & 28(71.8 \%)\end{array}$




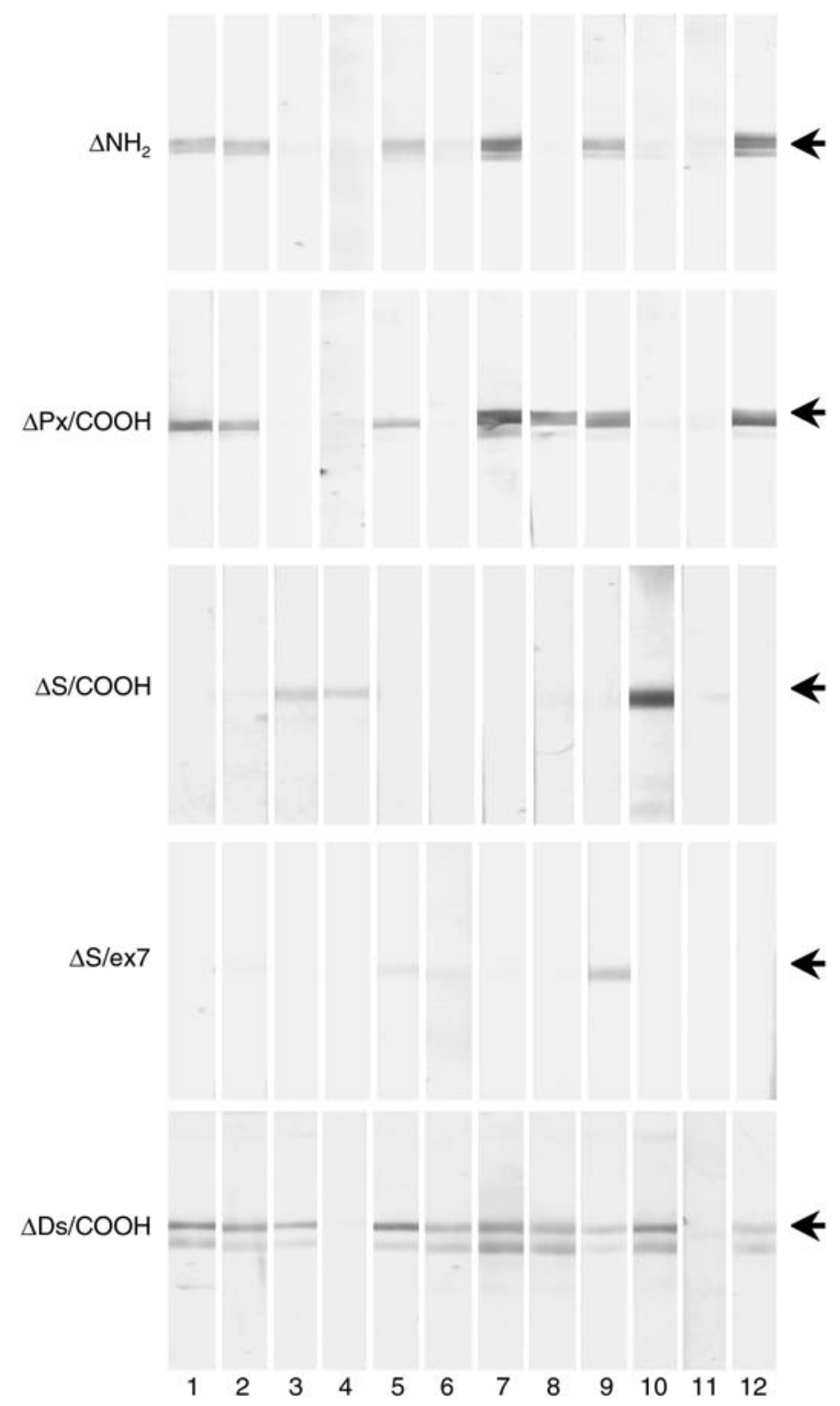

positive ECM1 reactivity. The difference between the lichen sclerosus group and these control subjects was statistically significant $(P<0.0001)$. For the other disease control subjects, 5 of 70 systemic lupus erythematosus (7.1\%; 95\% CI 1 to 13$), 6$ of 72 bullous pemphigoid (8.3\%; 95\% CI 2 to 15$)$, and 2 of 15 systemic sclerosis (13.3\%; $95 \%$ CI 0 to 27 ) were positive. Overall, the ECM1-ELISA gave 20 false-positive results in 318 control subjects (6.3\%), although these false-positive sera did not belong to any particular disease group. However, when the cutoff value was reassessed by use of an alternative standard formula (the mean OD unit of all the normal control subjects +3 SD , 0.473), 18 of 20 false-positive sera became negative, and only two sera from patients with systemic lupus erythematosus remained positive. Neither of these two sera showed any positive reactivity on ECM1 recombinant immunoblot or immunofluorescence microscopy on normal human skin (data not shown). Therefore, these false-positive results might be caused by nonspecific reactivity (e.g., against some minor contaminants from the antigen preparation or possibly from insufficient serum blocking).

\section{Figure 3}

Immunoblotting for lichen sclerosus sera by use of a series of recombinant ECM1-GST fusion fragments. The five recombinant ECM1-GST fragments, $\Delta \mathrm{NH}_{2}, \Delta \mathrm{Px} / \mathrm{COOH}, \Delta \mathrm{S} / \mathrm{COOH}, \Delta \mathrm{S} / \mathrm{ex} 7$, and $\Delta \mathrm{Ds} / \mathrm{COOH}$, were independently immunoblotted with serum samples from patients with lichen sclerosus (12 of which are illustrated) or control subjects. Each of the lichen sclerosus sera (numbered on the bottom) showed heterogeneous immunoreactivity against the recombinants. Note that $\Delta \mathrm{Ds} / \mathrm{COOH}$ represents the most antigenic epitope recognized by the vast majority of lichen sclerosus sera. Representative results from each recombinant blot are shown. Arrows indicate the positive signals.

ELISA values correlate with lichen sclerosus disease severity and duration. We analyzed the two clinical subgroups, severe disease $(n=17)$ or mild disease $(n=18)$, with individual ELISA scores against ECM1 (Figure 6). A significant correlation occurred between disease severity and ECM1-ELISA scores; patients with severe clinical phenotypes had higher ELISA scores compared with those who had mild disease (mean ELISA score \pm SD; $0.665 \pm 0.216$ vs. $0.425 \pm 0.129$, respectively; Mann-Whitney $U$ test, $P=0.031)$.

Interestingly, four of the severe-phenotype patients who suffered from lichen sclerosus complicated by squamous cell carcinoma on the affected skin (two of whom also had extragenital lichen sclerosus) exhibited higher ELISA scores overall $(0.689 \pm 0.230)$, although these scores did not differ significantly from the scores of other subjects in the severe-disease group without malignancy or with just genital skin involvement (Figure 6, filled circles and filled squares).

Apart from disease severity, the anti-ECM1-ELISA titers in these 35 patients and in four additional patients were assessed against disease duration (Table 3). Significantly lower ELISA scores occurred in the group who had the disease less than 1 year compared with any of the other three groups who had lichen sclerosus longer than 1 year. Higher ELISA scores were associated with patients who had longstanding disease, although there were no significant statistical differences between ELISA scores in any of the latter three groups; that is, in subjects who had lichen sclerosus 1 to 5 years, 5 to 10 years, or longer than 10 years.

Anti-ECM1 IgG from lichen sclerosus sera can access native ECM1 in vivo. Within 48 hours of the initial injection of each of the six affinity-purified IgG from lichen sclerosus sera, the ears of mice showed marked swelling with erythema. The swelling persisted, and, in addition, dilated blood vessels became evident within 12 days. These signs became most prominent at 20 days but persisted up to the final assessment point (28 days) (Figure 7A, left). In contrast, no swelling or changes in skin vasculature were noted after injection of control affinity-purified IgG into the mice ears (Figure 7A, right).

Histology of the affinity-purified IgG from lichen sclerosus sera-injected skin sites at 4 days and 28 days showed moderate inflammation with edema, patchy inflammatory cell infiltration, and dilated blood vessels (especially at 28 days) in the upper and mid-dermis (Figure 7B) in comparison to the skin of control subjects (Figure 7C). These changes are not specific, but are compatible with the early histological features seen in lichen sclerosus. However, no evidence of hyalinosis or scarring, typical histologic features of established (late) lichen sclerosus skin pathology, is present Moreover, immunohistologic examination revealed that 

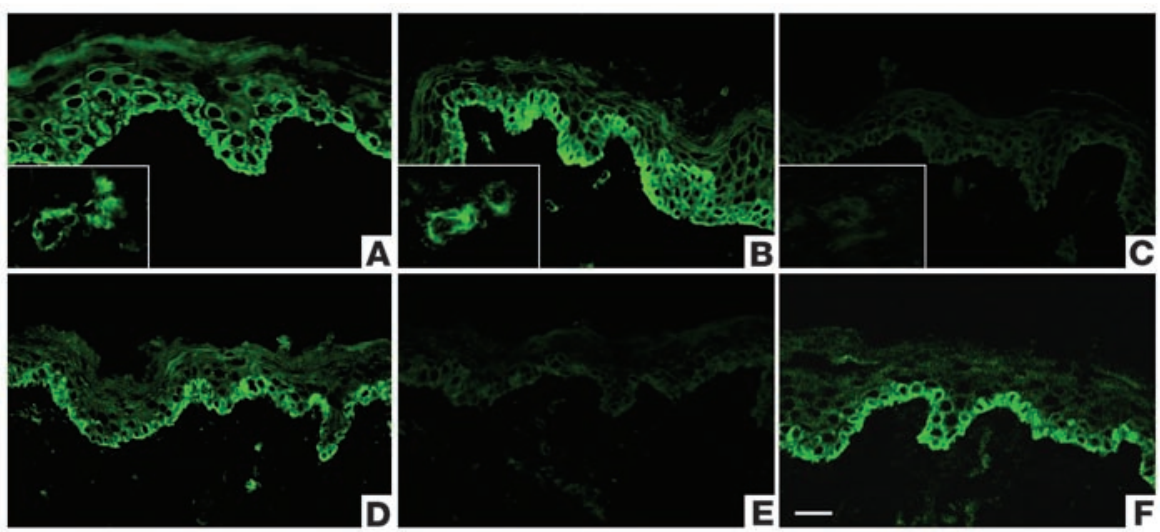

Figure 4

Preabsorption assays for affinity-purified lichen sclerosus sera with recombinant proteins. (A) Immunostaining with anti-ECM1 rabbit polyclonal antibody on normal human skin displays intracellular labeling in the lower epidermis, particularly in basal and suprabasal cell layers. Inset shows labeling of dermal blood vessels. (B) Similar staining pattern was obtained using affinitypurified IgG fractions from $\triangle \mathrm{Ds} / \mathrm{COOH}$-positive lichen sclerosus sera. (C) Before immunostaining, the affinity-purified IgG fractions from $\Delta \mathrm{Ds} / \mathrm{COOH}$-positive sera were incubated with an excess of $\Delta \mathrm{Ds} / \mathrm{COOH}$ recombinant. This results in a marked reduction in labeling intensity (c.f. Figure 4B). (D) Before immunostaining, the affinity-purified IgG fractions from a dual $\Delta \mathrm{NH}_{2}$-positive and $\Delta \mathrm{Ds} / \mathrm{COOH}$-positive lichen sclerosus serum were incubated with an excess of either $\Delta \mathrm{NH}_{2}$ or $\Delta \mathrm{Ds} / \mathrm{COOH}$ recombinants. No alteration occurred in the original labeling intensity. (E) Before immunostaining, the affinity-purified IgG fractions from a dual $\triangle \mathrm{NH}_{2}$-positive and $\triangle \mathrm{Ds} / \mathrm{COOH}$ positive sera were incubated with a mixture of both the $\Delta \mathrm{NH}_{2}$ and $\Delta \mathrm{Ds} / \mathrm{COOH}$ recombinants or with $(\mathbf{F})$ control recombinant protein BP180 NC16A. Note the marked signal reduction in $(E)$ but not in (F). Scale bar: $50 \mu \mathrm{m}$.

all six affinity-purified sera showed IgG deposition within the lower epidermis (intracellular) and surrounding dilated dermal blood vessels (Figure 7, D-F). These changes were very similar to those found in ECM1 immunolabeling of normal human skin and in mice injected with anti-ECM1 rabbit polyclonal antibody (data not shown). None of the other control IgG-injected mice had evidence of specific IgG deposition in their skin (Figure 7, G and $H)$. These findings suggest that lichen sclerosus IgG is capable of binding ECM1 in vivo.

\section{Discussion}

In this study, we have partially characterized the antigenic epitopes within the ECM1 protein that are detected by circulating
IgG autoantibodies in the sera of most patients with lichen sclerosus. We have also established a novel ELISA system that may be useful in providing a diagnostic marker of the disease and an indicator of clinical severity in affected individuals. Our large cohort study demonstrates that the ELISA using the recombinant protein that spans the distal second tandem repeat domain and $\mathrm{COOH}$-terminus of ECM1 (amino acids 359 to 559) is both highly sensitive and also highly specific. Moreover, the new ELISA method provides higher sensitivity and reliability for the detection of anti-ECM1 antibodies in lichen sclerosus sera than does immunoblotting when the full-length ECM1 recombinant protein is used (11).

Initial screening of the lichen sclerosus sera disclosed that the patients' autoantibodies recognize multiple epitopes within ECM1. More detailed assessment, such as selective preabsorption studies that use the recombinant fragment proteins, suggests that at least three major subsets of antigenic ECM1 epitopes are recognized by lichen sclerosus autoantibodies. These subsets are (a) epitopes that are reactive for both the $\mathrm{NH}_{2}$-terminal and $\mathrm{COOH}$-terminal proteins (b) epitopes that are restricted to the $\mathrm{COOH}$-terminus alone, and (c) epitopes that are restricted to just proximal to the $\mathrm{COOH}$ terminus. This finding means that screening with the recombinant protein that encompasses the distal second tandem repeat and the $\mathrm{COOH}$-terminal region covers almost the entire spectrum of antibody reactivity against ECM1 in lichen sclerosus sera.

In essence, our study has shown that antibodies to a recombinant ECM1 protein designed on amino acids 359 to 559 are detectable in the sera of most patients with lichen sclerosus. Furthermore, our passive-transfer experiments using neonatal mice clearly showed that affinity-purified lichen sclerosus IgG reactive with this domain can bind ECM1 in vivo and induce such skin inflammation as pronounced edema, mononuclear inflammatory cell

\section{Figure 5}

Quantitative analysis of IgG reactivity against the recombinant protein encompassing the distal $\mathrm{COOH}$-terminal region of ECM1 in the sera of lichen sclerosus patients. Sera from 95 patients with lichen sclerosus (LS) and from 318 comparative control subjects (161 normal volunteers, 70 with systemic lupus erythematosus [SLE], 72 with bullous pemphigoid [BP], 15 with systemic sclerosis [SSc]) were incubated with $\Delta \mathrm{Ds} / \mathrm{COOH}$ recombinant protein immobilized onto an ELISA plate. Bound IgG was detected with alkaline phosphatase-labeled anti-human IgG antibody. Each serum sample was run in duplicate. The mean OD values from the ECM1 recombinant protein were normalized by mean OD values of the background immunoreactivity (against GST control protein) and expressed as arbitrary units (AU) on the basis of the positive and negative reference sera. Dashed line indicates a cutoff value (0.328 AU), as determined by receiver operative characteristic (ROC) analysis (inset). The area under the curve, which represents the diagnostic accuracy of the ELISA, was 0.919 .

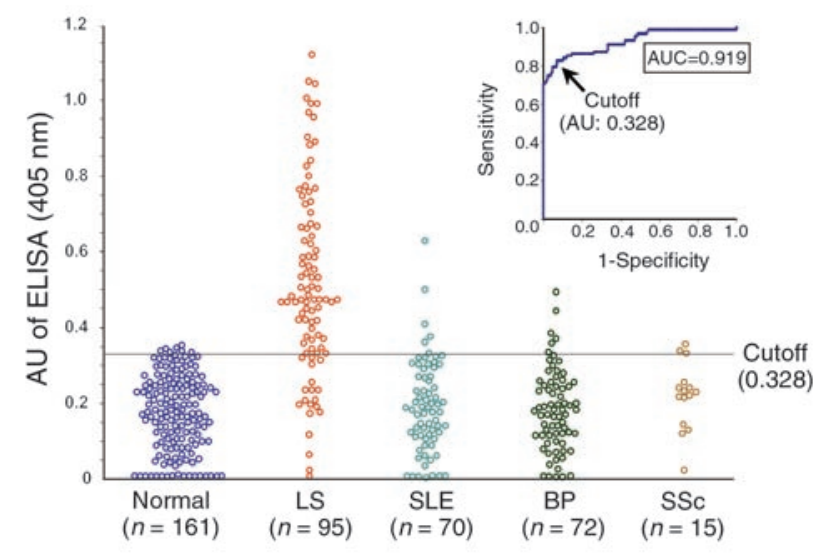


Table 2

Summary of ECM1-ELISA findings

\begin{tabular}{|c|c|c|c|c|c|}
\hline & \multicolumn{5}{|c|}{ Frequency of immunoreactivity ${ }^{A}$} \\
\hline & $n$ & ECM1 & $95 \% \mathrm{Cl}$ & $\begin{array}{l}\text { Comparison with } \\
\text { lichen sclerosus }\end{array}$ & $\begin{array}{c}\text { Comparison with healthy } \\
\text { control subjects }{ }^{A}\end{array}$ \\
\hline Lichen sclerosus & 95 & $76(80 \%)$ & $71 \%-88 \%$ & $\ldots$ & $P<0.0001$ \\
\hline $\begin{array}{l}\text { Healthy volunteers } \\
\text { Disease control }\end{array}$ & 161 & $7(4.3 \%)$ & $1 \%-7 \%$ & $P<0.0001$ & $\ldots$ \\
\hline Systemic lupus erythematosus & 70 & $5(7.1 \%)$ & $1 \%-13 \%$ & $P<0.0001$ & $P=0.88$ \\
\hline Bullous pemphigoid & 72 & $6(8.3 \%)$ & $2 \%-15 \%$ & $P<0.0001$ & $P=0.93$ \\
\hline Systemic sclerosis & 15 & $2(10.5 \%)$ & $0 \%-27 \%$ & $P<0.0001$ & $P=0.97$ \\
\hline
\end{tabular}

AThe cutoff value of the ELISA was determined by maximization of the Youden index.

mechanism, which may also help explain the predominant localization of lichen sclerosus to genital skin in humans.

Our study also highlighted some correlation between the titer of anti-ECM1 antibody, as detected by ELISA, and the clinical status of the patient. Specifically, patients with more severe clinical manifestations tended to have higher ELISA scores compared with those who had milder disease. More severe disease was

infiltration, and, significantly, dilated blood vessels in the dermis. These findings are consistent with the pathologic hallmark of early lesions in lichen sclerosus skin. However, passive transfer of the anti-ECM1 IgG did not induce hyalinosis or scarring in the mice. Possible reasons for this result include not only the time course of the experiment but also the structural and functional interspecies difference between human and murine ECM1 (12). Indeed, whereas functional knockout of ECM1 in human subjects results in lipoid proteinosis, attempts to make $E c m 1$ knockout mice have resulted in embryonic lethality (J. Merregaert, personal communication). In addition, if the function of ECM1 in human dermis and blood vessels is mainly to act as a "biological glue," for example binding to perlecan (13), a major heparan sulfate proteoglycan, or microassembly of other components of the extracellular matrix, such as interstitial collagen and elastic fibers or growth factors (16), then perhaps additional local factors such as trauma, moisture, infection, or, indeed, constitutive tissue damage from antigen-specific $\mathrm{T}$ cells might be required to develop the full histopathologic characteristics of lichen sclerosus $(17,18)$. As for the epidermal pathology, the demonstration of intracellular IgG deposition in the mice after passive transfer raises questions about whether IgG can pass transmembranously. In fact, several reports demonstrate penetration of IgG autoantibody into living cells $(19,20)$. Notably in keratinocytes, by using passive transfer and in vitro cell culture systems, a series of recent investigations have also demonstrated that IgG autoantibodies to desmoplakin I/II, entirely intracellular desmosomal antigens, can get into living cells and reach the target antigens (21-23). Moreover, anti-nuclear IgG antibodies from patients with systemic lupus erythematosus have been shown to penetrate into living epithelial cells via receptor-mediated endocytosis and subsequently localize to the corresponding intracellular target antigens (24). Considering these findings, several mechanisms might be implicated in the internalization of anti-ECM1 IgG from lichen sclerosus patients; for example (a) concentrationdependent passive transport (b) cell surface receptor-mediated positive transport, or (c) a combination of these. Aside from these hypotheses, the inflammatory reaction induced by the anti-ECM1 IgG is possibly capable of producing sufficient epidermal damage that then allows autoantibody access to intracellular ECM1, followed by antibody-antigen binding. Thus, although we cannot conclusively define the mechanism of the intracellular in vivo IgG deposition for ECM1, the literature contains substantial information to justify our data. Clearly, however, further investigations are needed to develop a more complete understanding of disease usually associated with longer duration of disease, but correlation between ELISA score and length of disease was only noted in patients with lichen sclerosus that lasted less than 1 year compared with disease present for more than 1 year. Nevertheless, longitudinal studies that record both disease activity and ECM1 antibody titers over time in the same individual are necessary to address this problem further. Likewise, whether the development of multiple antigenic targets within ECM1 has any pathogenic significance or whether it merely represents a secondary phenomenon occurring within the setting of more severe and chronic disease remains to be seen. One possible explanation for these multiple epitopes might be the phenomenon of "epitope spreading," which is frequently seen in other autoimmune skin disorders (25). Notably, during the course of an autoimmune condition, patient autoreac-

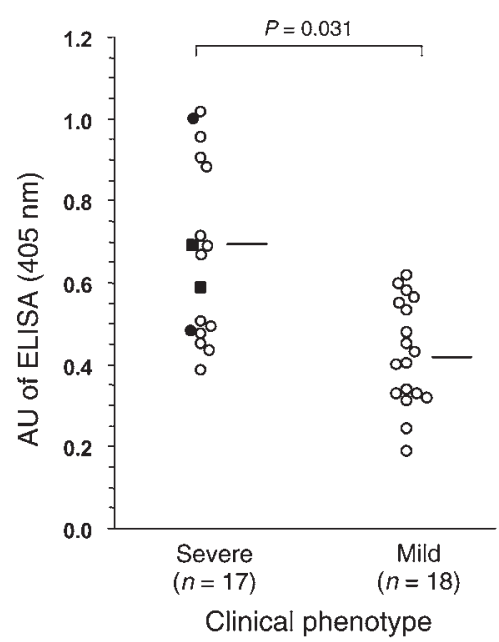

\section{Figure 6}

Comparison between ECM1-ELISA values and clinical presentation of lichen sclerosus patients. Disease severity of 35 well-defined patients with lichen sclerosus was divided into two clinical subgroups, "severe" and "mild," and analyzed with individual ELISA scores against ECM1. The ECM1-ELISA scores were significantly higher in patients with a severe clinical phenotype compared with those with a mild phenotype (mean ELISA score \pm SD; $0.665 \pm 0.216$ vs. $0.425 \pm 0.129$, respectively; Mann-Whitney $U$ test, $P=0.031$ ). Horizontal bars indicate the mean ELISA values in each subgroup. In the severe group, four cases had longstanding disease complicated by genital skin squamous cell carcinoma; two of whom had just genital disease (filled circles), whereas two others had both genital and extragenital involvement (filled squares). 


\section{Table 3}

Comparison between the ECM1-ELISA scores and lichen sclerosus disease duration

\begin{tabular}{lccc}
$\boldsymbol{n}$ & Duration (y) & Average $(\mathbf{y})$ & Mean ELISA score $(\mathbf{A U})^{\mathbf{A}} \pm \mathbf{S D}$ \\
5 & $<1$ & 0.8 & $0.377 \pm 0.115$ \\
18 & $1-5$ & 3.5 & $0.612 \pm 0.196^{\mathrm{B}}$ \\
5 & $5-10$ & 9.0 & $0.501 \pm 0.305$ \\
11 & $>10$ & 19.1 & $0.577 \pm 0.208^{\mathrm{B}}$ \\
\hline
\end{tabular}

AThe ELISA scores were expressed in arbitrary units $(A U)$ based on the positive and negative reference sera, which were set to "1.0" and "O" OD units, respectively. ${ }^{\mathrm{B} C o m p a r i s o n}$ with the shortest disease duration group $(x \leq 1$ y) revealed a statistical significance (MannWhitney $U$ test, $P<0.05)$.

tive $\mathrm{T}$ cell and $\mathrm{B}$ cell responses do not always restrict to a unique immunodominant epitope, but may extend to include additional secondary epitopes within the same antigen.

In this study, we extended our previous observations that detection of anti-ECM1 antibodies in lichen sclerosus may serve as a useful diagnostic marker (11). Unfortunately, the antibodies are present at titers too low to be detected by conventional indirect immunofluorescence microscopy, as routinely performed for the diagnosis of such autoimmune skin blistering/scarring diseases as pemphigus and bullous pemphigoid (26). For lichen sclerosus sera or skin, immunofluorescence microscopy is usually negative or only faintly positive. Indeed, most other related studies have been unable to detect in vivo bound antibodies (apart from fibrin) in involved skin (27). The anti-ECM1 antibodies can be detected by indirect immunofluorescence microscopy after affinity purification (11), but such an approach is not practical for routine diagnostic laboratories. Likewise, the anti-ECM1 autoantibodies can be detected by immunoblotting against epidermal extracts, but again, this technique is not ideally suited to routine laboratory activity. The novel ECM1-ELISA system now offers a practicable alternative means of assessment that is not only rapid but also

\section{Figure 7}

Passive-transfer study using affinity-purified lichen sclerosus IgG in neonatal BALB/c mice. (A) Intradermal injection of affinity-purified lichen sclerosus IgG into BALB/c mouse caused extensive erythematous swelling with telangiectasie (left ear), whereas the control skin injected with either nonimmune human IgG exhibited no evidence of skin inflammation (right ear). This picture was taken at 28 days in a mouse that had received seven separate injections in both ears at days $0,4,8,12,16,20$, and 24 . (B) Light microscopy of the mouse skin injected with affinity-purified lichen sclerosus IgG exhibited a pronounced inflammatory infiltration with edema and dilated blood vessels in the upper-middle dermis. The overlying epidermis showed mild acanthosis. (C) In contrast, the control skin injected with affinity-purified normal human IgG showed only a scanty perivascular infiltration in the dermis. (D) The mouse skin injected with affinity-purified lichen sclerosus antibodies revealed IgG deposition within the lower epidermis (arrows) and surrounding dilated dermal blood vessels (arrowheads). Asterisk indicates the injected site. Higher magnification views revealed an intracellular signal in basal keratinocytes and weaker staining in the suprabasal keratinocytes $(\mathbf{E})$, as well as in the walls of dilated dermal blood vessels (F). (G) In contrast, the control injection with affinity-purified normal human IgG resulted in no immunolabeling. (H) At higher magnification, control injections only resulted in a nonspecific signal in the corneal layers. Scale bar: $50 \mu \mathrm{m}$. more sensitive than immunoblotting. Similar technical developments of diagnostic ELISAs have already become established for autoimmune skin diseases such as pemphigus (28), and now determination of whether the ECM1-ELISA will prove equally useful in the diagnosis, and perhaps monitoring, of patients with lichen sclerosus has become possible.

Our new data linking ECM1-ELISA titer to clinical severity may also provide a rationale for the development of new treatment options for patients with lichen sclerosus that is unresponsive to standard regimens such as potent topical corticosteroids or systemic immunosuppressants. For example, immunoadsorption therapy that utilizes the particular recombinant antigen could be valuable in efficiently removing circulating anti-ECM1 antibodies from the lichen sclerosus patients' sera. Because this treatment is target specific, it may reduce potentially harmful and unpleasant side effects associated with the use of such systemic agents as corticosteroids, retinoids, and cyclosporin $(3,6,29,30)$ and may lessen the need for surgical intervention for complications of scarring or malignancy $(18,31)$.

In summary, this study provides the first evidence that lichen sclerosus IgG autoantibodies to the ECM1 glycoprotein may directly contribute to the pathophysiology of the disease. Moreover, our novel ELISA is useful in quantifying autoantibody reactivity, which potentially reflects the clinical status of patients with lichen sclerosus. In addition to routine clinical and skin biopsy examination, we
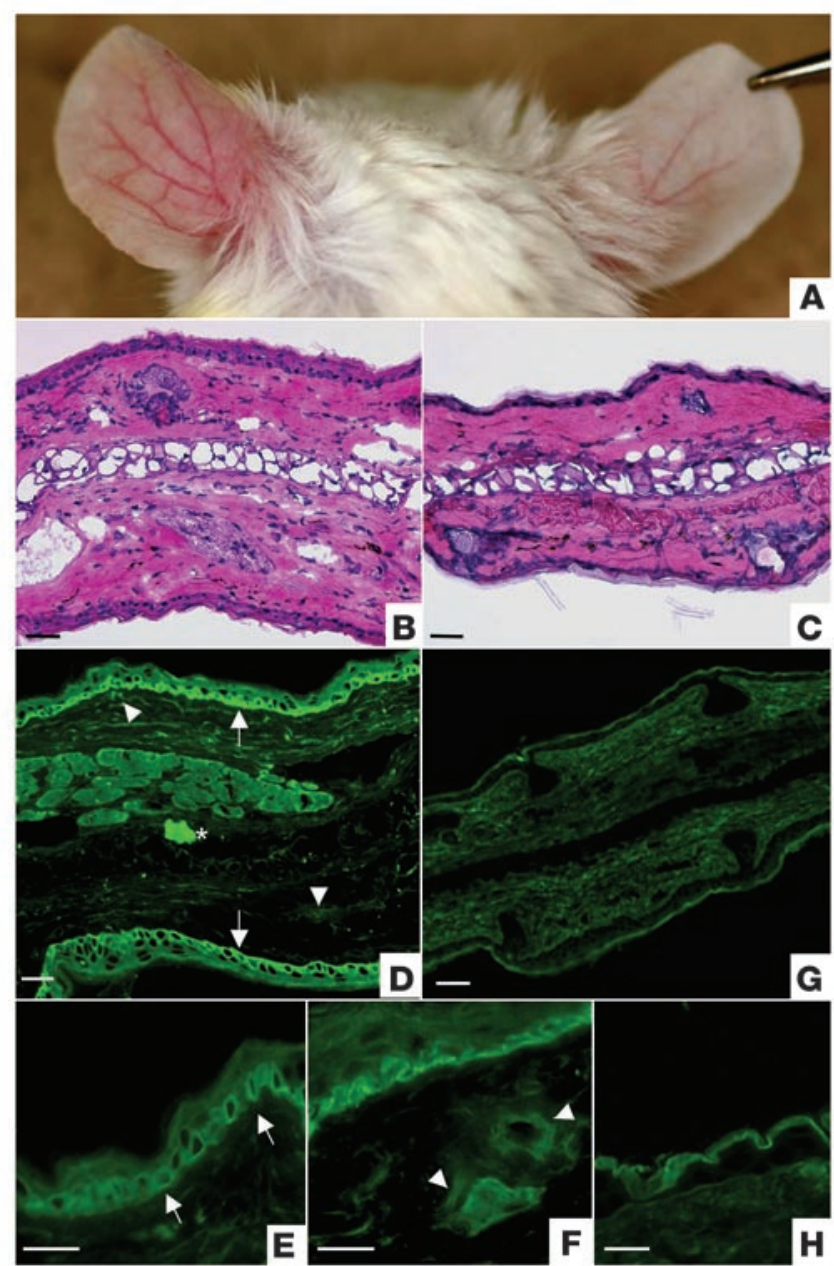
believe that this simple ELISA assay may be helpful in the diagnosis and management of patients with lichen sclerosus.

\section{Methods}

Patient details. We assessed serum samples from 95 patients with lichen sclerosus (93 female and 2 male) and 161 healthy age/sexmatched control subjects with no history of autoimmune diseases. The lichen sclerosus subjects ranged from 26 to 88 years of age. All had genital disease but seven also had extragenital involvement. The clinical diagnosis was confirmed histologically in more than $70 \%$ of patients. All patients had no clinical overlap with morphea or other autoimmune diseases, although four subjects had thyroiditis, of whom one also had vitiligo and pernicious anemia, and one other had vitiligo. Nearly all patients were applying topical potent or ultrapotent steroid ointments to their lichen sclerosus skin at the time of serum sampling. Of the lichen sclerosus sera, 64/95 samples had been shown previously to react with full-length ECM1 recombinant protein on immunoblotting (11). For clinical evaluation, detailed information was available from 35 lichen sclerosus patients, who were then classified as either "severe" or "mild." Given the lack of published criteria for determining the actual clinical severity of lichen sclerosus, the severe group $(n=17)$ comprised those patients with disease complicated by genital skin squamous cell carcinoma $(n=4)$, two of whom had extragenital as well as genital lichen sclerosus, and 13 other patients with either active enlarging skin plaques or disease that was difficult to control by application of topical steroids or that frequently relapsed. The mild group $(n=18)$ comprised patients with genital lichen sclerosus whose condition was adequately controlled by topical steroids or in whom the disease was inactive. Unfortunately, the cohort did not include any patients with just extragenital lichen sclerosus. In addition, apart from the disease severity that was the basis of two groups, disease duration was used to divide the patients into four groups consisting of those who had the disease less than 1 year, 1 to 5 years, 5 to 10 years, and more than 10 years. All clinical information was accurately recorded at the same time as the sera were taken.

As disease controls, archival serum samples were chosen randomly from 157 subjects with other autoimmune sclerosing or basement-membrane diseases. These samples comprised systemic sclerosis $(n=15)$, systemic lupus erythematosus $(n=70)$, and bullous pemphigoid $(n=72)$. These control diseases were diagnosed by clinical criteria, routine histology, and immunopathology.

All serum samples were collected from patients attending outpatient clinics at St. Thomas' Hospital (London, United Kingdom), Churchill Hospital (Oxford, United Kingdom), Ashford Hospital (Ashford, United Kingdom), and St, Peter's Hospital (Chertsey, United Kingdom). Samples were anonymized and the underlying diagnosis was concealed from the investigators performing the experiments. Local ethics committee approval was obtained for all parts of the study. All serum samples were stored at $20^{\circ} \mathrm{C}$ until assayed.

DNA. The nucleotide and amino acid sequences of human ECM1 cDNA were based on previously published data (13).

Construction of ECM1 cDNA fragments. We have recently constructed an E. coli transfer vector that contains the full-length 1.8$\mathrm{kb}$ ECM1 cDNA (11). This construct was used to make a series of truncated recombinant fragments that spanned almost the entire ECM1 sequence (see Figure 1).

To generate the $\Delta \mathrm{NH}_{2}$ clone, the original full-length ECM1 vector was digested with PvuII, and the excised cDNA fragment corre- sponding to the $\mathrm{NH}_{2}$-terminal region (nucleotides 94 to 615) was ligated in-frame into the prokaryotic GST fusion vector, pGEX4T-3 (Amersham Pharmacia Biotech, Buckinghamshire, United Kingdom), at SmaI restriction sites, with correct orientation.

For construction of clones $\Delta \mathrm{Px} / \mathrm{COOH}$ and $\Delta \mathrm{S} / \mathrm{COOH}$, the original ECM1 vector was first digested with $B s a \mathrm{I}$, which resulted in generation of two cDNA fragments, $\mathrm{Px} / \mathrm{COOH}$ (nucleotides 616 to 1438 ) and S/COOH (nucleotides 1439 to 1709). After blunt-ended digestion with Klenow enzyme, these fragments were subcloned independently into pBlueScript vector (Stratagene, La Jolla, California, USA) at an EcoRV site. The Px/COOH fragment was then inserted in-frame into pGEX-4T-3 vector at SalI and Not I sites, whereas the $\mathrm{S} / \mathrm{COOH}$ fragment was introduced into the pGEX-4T-2 vector at EcoRI and NotI sites.

For construction of the $\Delta \mathrm{S} / \mathrm{ex} 7$ clone, the $\mathrm{Px} / \mathrm{COOH}$ fragment obtained above was excised with BamHI and PvuII, blunt-ended, and inserted with correct orientation into the pGEX-4T-3 vector at a SmaI site.

The $\Delta \mathrm{Ds} / \mathrm{COOH}$ clone was prepared by digesting the original full-length ECM1 vector with PvuII and NotI, followed by relegating in-frame into the pGEX-4T-2 vector at SmaI and NotI sites.

These procedures all fuse the GST tag at the $\mathrm{NH}_{2}$-terminus of the recombinant proteins. All the cDNA constructs were sequenced to confirm the identity and fidelity of the subcloning steps.

Induction of recombinant protein. The ECM1 cDNA clones obtained above were transformed independently into E. coli strain BL21Star (DE3) (Invitrogen, Groningen, The Netherlands) and inoculated into liter cultures of 2xYT medium containing $50 \mu \mathrm{g} / \mathrm{ml}$ ampicillin, followed by protein induction using 0.5 to $1 \mathrm{mM}$ isopropyl $\beta$-D-thiogalactopyranoside (Amersham Pharmacia Biotech). After the protein extraction, the recovered recombinant protein was purified by affinity chromatography using a glutathione sepharose 4B column (Amersham Pharmacia Biotech). The protein concentration was determined by Bradford protein assay (Bio-Rad, Hercules, California, USA).

Affinity purification and preabsorption of patients' sera. IgG fractions from lichen sclerosus patients' sera (selected on the basis of positive recombinant ECM1 protein immunoblots) were affinity purified using Protein G-Sepharose columns (Amersham Pharmacia Biotech). After washing with ice-cold PBS (pH7.5), bound IgGs were eluted with $0.1 \mathrm{~mol} / 1$ glycine $(\mathrm{pH} 2.5)$ and neutralized immediately with $1 \mathrm{~mol} / 1 \mathrm{Tris} / \mathrm{HCl}$ ( $\mathrm{pH} 9.0)$. The fractionated samples were dialyzed with PBS at $4^{\circ} \mathrm{C}$ overnight and concentrated by Centricon-10 columns (Amicon, Gloucestershire, United Kingdom). The resultant IgGs were incubated overnight at $4^{\circ} \mathrm{C}$ with an equal amount of either the recombinant ECM1 proteins or the control proteins, GST or BP180 NC16a (the latter representing the antigenic epitope most frequently recognized in sera from patients with the common autoimmune blistering skin disease, bullous pemphigoid) (32), followed by precipitation. IgG fractions from each of three normal human subjects were prepared in a similar manner and used for control experiments. The supernatants were collected and tested for any residual immunoreactivity on normal human skin sections and ELISA.

Immunohistochemistry. Indirect immunofluorescence was performed using standard methods, as described previously (33). Briefly, $5-\mu \mathrm{m}$ frozen sections of normal human skin were incubated at $37^{\circ} \mathrm{C}$ for $1 \mathrm{~h}$ with affinity-purified IgGs from patients' sera (1:100 dilution in PBS), control IgG from normal human sera (1:100 dilution in PBS), or anti-ECM1 rabbit polyclonal antibody 
in combination with or without the preabsorption step, followed by FITC-labeled anti-human IgG antibody (Dako, Cambridgeshire, United Kingdom). Details of the ECM1 antibody (raised against the COOH-terminal 17 amino acids of human ECM1) have been published elsewhere (11).

Immunoblotting. The ECM1-GST fusion proteins were separated on $12 \%$ nongradient SDS-polyacrylamide gels and visualized by Coomassie blue staining or immunoblotting with horseradish peroxidase-labeled rabbit anti-GST antibody (Amersham Pharmacia Biotech), to verify molecular weight and quality of the protein purification.

For serum immunoblotting, equivalent amounts of each recombinant ECM1 protein loaded on the gels were transblotted onto nitrocellulose membranes (Bio-Rad). After blocking with Trisbuffered saline (TBS) $/ 3 \%$ skimmed milk, the membranes were cut into small strips and incubated overnight at $4{ }^{\circ} \mathrm{C}$ with the patients' sera (1:20 dilution with TBS/3\% skim milk). The membrane strips were then reacted with alkaline phosphatase-labeled secondary antibodies (Dako), followed by signal development using nitroblue tetrazolium substrate (Bio-Rad).

ECM1 ELISA. Optimal conditions for the ELISA method were established by various chessboard titrations following technical protocols published elsewhere (34).

The recombinant proteins were immobilized on 96-well microtiter plates (MaxiSorp; Nunc, Roskilde, Denmark) by coating each well with either $0.5 \mu \mathrm{g}$ of $\Delta \mathrm{Ds} / \mathrm{COOH}$ or a molar equivalent amount of the control GST protein in $100 \mu \mathrm{l}$ of $0.1 \mathrm{M}$ carbonatebicarbonate buffer ( $\mathrm{pH} 9.8$ ) at $4^{\circ} \mathrm{C}$ overnight. All procedures were performed at room temperature, except the antigen coating. After each step, the wells were washed four times with $50 \mathrm{mM}$ Tris/ $\mathrm{HCl}$ ( $\mathrm{pH} 7.5$ ) containing $0.05 \%$ Tween 20 (TBS-T). The ELISA plates were incubated for $2 \mathrm{~h}$ with $200 \mu \mathrm{l}$ of a blocking buffer consisting of $3 \%$ bovine serum albumin (fraction V; Sigma-Aldrich, St. Louis, Missouri, USA) in TBS-T. Sera were diluted 1:100 in the blocking buffer, and then added to each well in duplicate. After incubating for $2 \mathrm{~h}$, the bound IgG were detected with $100 \mu \mathrm{l}$ of alkaline phosphatase-conjugated anti-human IgG rabbit $\mathrm{F}\left(\mathrm{ab}^{\prime}\right)_{2}$ polyclonal antibody (Dako) diluted 1:4,000 in TBS-T, followed by colorimetric assay using $p$-nitrophenylphosphate (Sigma-Aldrich). The color development was stopped by $3 \mathrm{M} \mathrm{NaOH}$. The optical density (OD) was measured at $405 \mathrm{~nm}$ by an automated plate reader (Revelation; Dynex Technologies, Chantilly, Virginia, USA) according to kinetic measurements so that the ODs of a positive reference sera or anti-ECM1 antibody reached at least "1.0." In each serum sample, the background reactivity against the control GST protein was subtracted from the mean OD values with $\Delta \mathrm{Ds} /$ $\mathrm{COOH}$. The antibody concentrations obtained were expressed in
AUs based on the positive and negative reference sera, which were set to " 1.0 " and " 0 " OD units, respectively. The reference sera and anti-ECM1 antibody were always used to correct interplate/intraplate variability for this assay.

Statistics. Statistical analysis was performed using SPSS version 11.0 (SPSS, Chicago, Illinois, USA). ROC curves were used to evaluate the ability of the ELISA to detect specific autoantibodies against the $\Delta \mathrm{Ds} / \mathrm{COOH}$ recombinant protein. The AUC index was reported with the relative $95 \%$ CI to compare ECM1 seroreactivity between lichen sclerosus and all comparative control groups. The Youden index was used to select the best cutoff values for the ELISA. To assess the reproducibility of the ELISA results, coefficients of variance were calculated for reference lichen sclerosus and normal control sera. Comparison of the ELISA results between patient and control groups was statistically analyzed using MannWhitney's nonparametric, unpaired, two-tailed test and Pearson's correction test or Fisher's exact probability test when more than $20 \%$ of the expected values were lower than 5 .

Passive-transfer study. Neonatal BALB/c mice (age, 24 to 36 h; body weight, 1.4 to $1.8 \mathrm{~g}$ ) were used for passive-transfer experiments (32, 35). The ears of the mice were injected intradermally with either (a) affinity-purified IgG from any one of six lichen sclerosus patients (b) affinity-purified IgG from any of three normal human subjects (c) PBS, or (d) anti-ECM1 polyclonal antibody. In individual mice, the left and right ear was injected with a different solution. For the IgG injections, the amount injected was 0.5 to $1.0 \mathrm{mg}$ of total IgG per $\mathrm{g}$ of body weight. Injections were given every 4 days up to 24 days. All mice were physically examined daily and skin biopsies were taken at day 0 , day 4, and day 28 . Each mouse only underwent one biopsy from each ear. The total number of mice used for these experiments was 27 . All skin samples were examined by light microscopy (H\&E staining) and by immunofluorescence microscopy for IgG deposition.

\section{Acknowledgments}

This work was funded by grants from the Charitable Foundation of Guy's and St. Thomas' Hospitals, the British Skin Foundation, and Smith's Charity, London, United Kingdom.

Received for publication October 23, 2003, and accepted in revised form March 30, 2004.

Address correspondence to: John A. McGrath, Genetic Skin Disease Group, St. John's Institute of Dermatology, St. Thomas' Hospital, Lambeth Palace Road, London SE1 7EH, United Kingdom. Phone: 44-20-7928-9292 ext. 3315; Fax: 44-20-7922-8175; E-mail: john.mcgrath@kcl.ac.uk.
1. Meffert, J.J., Davis, B.M., and Grimwood, R.E. 1995. Lichen sclerosus. J. Am. Acad. Dermatol. 32:393-416.

2. Tasker, G.L., and Wojnarowska, F. 2003. Lichen sclerosus. Clin. Exp. Dermatol. 28:128-133.

3. Powell, J.J., and Wojnarowska, F. 1999. Lichen sclerosus. Lancet. 353:1777-1783.

4. Meyrick-Thomas, R.H., Ridley, C.M., McGibbon, D.H., and Black, M.M. 1998. Lichen sclerosus et atrophicus and autoimmunity-a study of 350 women. Br. J. Dermatol. 118:41-46.

5. Wallace, H.J. 1971. Lichen sclerosus et atrophicus. Trans. St. Johns. Hosp. Dermatol. Soc. 57:9-30.

6. Neill, S.M., Tatnall, F.M., and Cox, N.H. 2002. British Association of Dermatologists: guidelines for the management of lichen sclerosus. Br. J. Dermatol.
147:640-649

7. Azurdia, R.M., et al. 1999. Lichen sclerosus in adult men: a study of HLA associations and susceptibility to autoimmune disease. Br. J. Dermatol. 140:79-83.

8. Powell, J., Wojnarowska, F., Winsey, S., Marren, P., and Welsh, K. 2000. Lichen sclerosus premenarche: autoimmunity and immunogenetics. $\mathrm{Br}$. J. Dermatol. 142:481-484.

9. Harrington, C.I., and Dunsmore, I.R. 1981. An investigation into the incidence of auto-immune disorders in patients with lichen sclerosus and atrophicus. Br. J. Dermatol. 104:563-566.

10. Irvine, H.G. 1913. Idiopathic atrophy of the skin. JAMA. 61:396-400.

11. Oyama, N., et al. 2003. Autoantibodies to extracellular matrix protein 1 in lichen sclerosus.
Lancet. 362:118-123.

12. Smits, P., et al. 1997. The human extracellular matrix gene 1 (ECM1): genomic structure, cDNA cloning, expression pattern, and chromosomal localization. Genomics. 45:487-495.

13. Mongiat, M., et al. 2003. Perlecan protein core interacts with extracellular matrix protein 1 (ECM1), a glycoprotein involved in bone formation and angiogenesis. J. Biol. Chem. 278:17491-17499.

14. Hamada, T., et al. 2002. Lipoid proteinosis maps to $1 \mathrm{q} 21$ and is caused by mutations in the extracellular matrix protein 1 gene (ECM1). Hum. Mol. Genet. 11:833-840.

15. Hamada, T. 2002. Lipoid proteinosis. Clin. Exp. Dermatol. 27:624-629.

16. Chan, I. 2004. The role of extracellular matrix pro- 
tein 1 in human skin. Clin. Exp. Dermatol. 29:52-56. 17. Chan, I., et al. 2004. Characterisation of IgG autoantibodies to extracellular matrix protein 1 (ECM1) in lichen sclerosus. Clin. Exp. Dermatol. In press.

18. Neill, S.M., and Ridley, C.M. 2001. Management of anogenital lichen sclerosus. Clin. Exp. Dermatol. 26:637-643.

19. Galoppin, L., and Saurat, J.H. 1981. In vitro study of the binding of antiribonucleoprotein antibodies to the nucleus of isolated living keratinocytes. J. Invest. Dermatol. 76:264-267.

20. Alarcon-Segovia, D., Ruiz-Arguelles, A., and Llorente, L. 1996. Broken dogma: penetration of autoantibodies into living cells. Immunol. Today. 17:163-164.

21. Foedinger, D., et al. 1995. Autoantibodies to desmoplakin I and II in patients with erythema multiforme. J. Exp. Med. 181:169-179.

22. Foedinger, D., et al. 1996. Autoantibodies against desmoplakin I and II define a subset of patients with erythema multiforme major. J. Invest. Dermatol. 106:1012-1016.

23. Foedinger, D., et al. 1998. Erythema multiforme associated human autoantibodies against desmoplakin I and II: biochemical characterization and passive transfer studies into newborn mice. J. Invest. Dermatol. 111:503-510.

24. Golan, T.D., Gharavi, A.E., and Elkon, K.B. 1993. Penetration of autoantibodies into living epithelial cells. J. Invest. Dermatol. 100:316-322.

25. Chan, L.S., et al. 1998. Epitope spreading: lessons from autoimmune skin diseases. J. Invest. Dermatol. 110:103-109.

26. Katz, S.I., Halprin, K.M., and Inderbitzin, T.M. 1969. The use of human skin for the detection of anti-epithelial autoantibodies: a diagnostic and prognostic test. J. Invest. Dermatol. 53:390-399.

27. Bushkell, L.L., Friedrich, E.G., Jr., and Jordon, R.E. 1981. An appraisal of routine direct immunofluorescence in vulvar disorders. Acta. Derm. Venereol. 61:157-161.

28. Ishii, K., et al. 1997. Characterization of antoantibodies in pemphigus using antigen-specific enzyme-linked immunosorbent assays with baculovirus-expressed recombinant desmogleins. J. Immunol. 159:2010-2017.

29. Bousema, M.T., et al. 1994. Acitretin in the treatment of severe lichen sclerosus et atrophicus of the vulva: a double-blind, placebo-controlled study. J. Am. Acad. Dermatol. 30:225-231.

30. Smith, Y.R., and Haefner, H.K. 2004. Vulvar lichen sclerosus: pathophysiology and treatment. Am. J. Clin.Dermatol. 5:105-125.

31. Campus, G.V., Ena, P., and Scuderi, N. 1984. Surgical treatment of balanitis xerotica obliterans. Plast. Reconst. Surg. 73:652-657.

32. Liu, Z., et al. 1993. A passive transfer model of the organ-specific autoimmune disease, bullous pemphigoid, using antibodies generated against the hemidesmosomal antigen, BP180. J. Clin. Invest. 92:2480-2488.

33. Black, M.M., and Bhogal, B.S. 1990. Diagnosis, diagnostic and research techniques. In Management of blistering diseases. R.A.Briggaman and F. Wojnarowska, editors Raven Press. New York, New York, USA. 15-34.

34. Kemeny, D.M., Price, J.F., Richardson, V., Richards, D., and Lessof, M.H. 1991. The IgE and IgG subclass antibody response to foods in babies during the first year of life and their relationship to feeding regimen and the development of food allergy. J. Allergy. Clin. Immunol. 87:920-929.

35. Borradori, L., et al. 1995. Passive transfer of autoantibodies from a patient with mutilating epidermolysis bullosa acquisita induces specific alterations in the skin of neonatal mice. Arch. Dermatol. 131:590-595. 